Christoph Strümpfel, Eric Schuster, Svenja Huschbeck, Christian Berth, Maarten Uijt De Haag

\title{
Assured Multi-Mode Navigation for Urban Operations of Small UAS
}

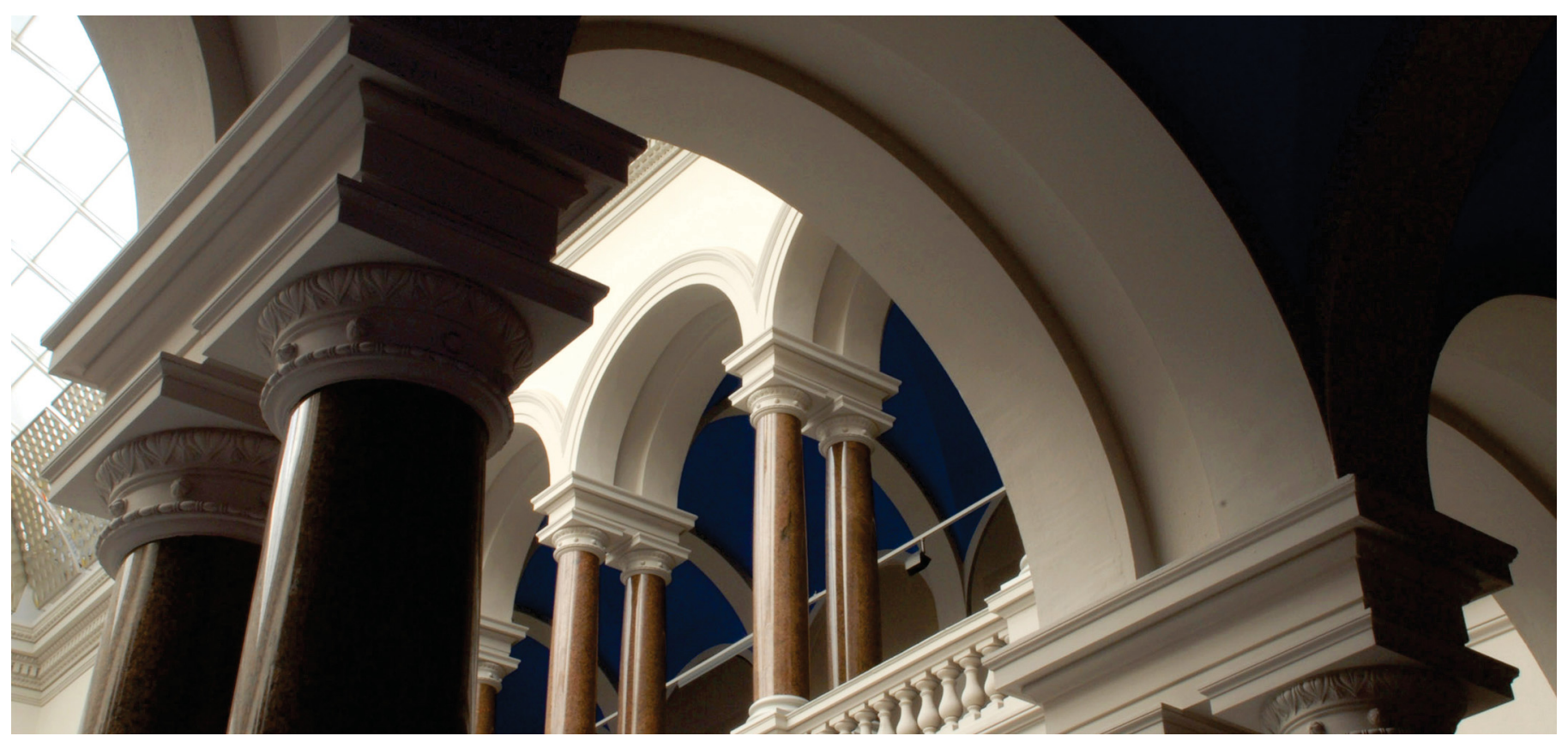

Strümpfel, C., Schuster, E., Huschbeck, S., Berth, C., \& Uijt De Haag, M. (2020). Assured Multi-Mode Navigation for Urban Operations of Small UAS. In: AIAA Scitech 2020 Forum. https://doi. org/10.2514/6.2020-1945 


\title{
Assured Multi-Mode Navigation for Urban Operations of Small UAS
}

\author{
Christoph Strümpfel ${ }^{1}$, Eric Schuster ${ }^{1}$, Svenja Huschbeck ${ }^{1}$, Christian Berth ${ }^{2}$ and \\ Maarten Uijt de $\mathrm{Haag}^{3}$ \\ Technische Universität Berlin
}

This paper was first published in the proceedings of the AIAA SciTech Forum 2020 in Orlando and does not show any changes in content to the primary publication. The full reference to the first publication is:

Struempfel, C., Schuster, E., Huschbeck, S., Berth, C., \& Uijt De Haag, M. (2020). Assured Multi-Mode Navigation for Urban Operations of Small UAS. AIAA Scitech 2020 Forum. https://doi.org/10.2514/6.2020$\underline{1945}$

\begin{abstract}
The paper discusses the preliminary analysis of an urban availability tool required for preflight flight planning. In this tool, areas in an urban environment are assessed with respect to GNSS availability, separation to traffic and obstacles, impact of UAS noise, and various other criteria. GNSS availability will drive the necessity to include alternative navigation sensors such as laser scanners, vision sensors and altimeters in the navigation mechanization to meet the required navigation performance necessary for urban operations. This paper shows the initial results of the GNSS availability tool and discusses the sensor integration strategy when GNSS-only availability is not sufficient.
\end{abstract}

\section{NOMENCLATURE}

$\begin{array}{lll}\text { ADS-B } & =\text { Automatic Dependent Surveillance }- \text { Broadcast } \\ \text { DOP } & =\text { Dilution of Precision } \\ \text { GML } & =\text { Geography Markup Language } \\ \text { GNSS } & =\text { Global Navigation Satellite Systems } \\ \text { GPS } & =\text { Global Positioning System } \\ \text { HDOP } & =\text { Horizontal Dilution of Precision } \\ \text { HPL } & =\text { Horizontal Protection Level } \\ \text { PBN }= & \text { Performance Based Navigation } \\ \text { SLAM }= & \text { Simultaneous Localization and Mapping } \\ \text { TMA }= & \text { Terminal Maneuvering Areas } \\ \text { UAS }=\text { Unmanned Aircraft System } \\ \text { UTM }=\text { UAS Traffic management } \\ \text { VDOP }= & \text { Vertical Dilution of Precision } \\ \text { VPL }= & \text { Vertical Protection Level }\end{array}$

1 Research Assistant and PhD candidate, Chair of Flight Guidance \& Air Transport, TU Berlin.

2 Research Engineer and flight test pilot, Chair of Flight Guidance \& Air Transport, TU Berlin.

3 Professor of Chair, Chair of Flight Guidance \& Air Transport, TU Berlin, AIAA Associate Fellow. 


\section{INTRODUCTION}

For many commercial UAS, Global Navigation Satellite Systems (GNSS) such as the Global Positioning System (GPS) have become one of the most dependable solutions for position and navigation, however, in some of the operational environments like the urban environment shown in Fig. 1 (left), GNSS may be unavailable or only sparsely available due to shadowing, significant signal attenuation, multipath, or even intentional denial or deception. For example, sUAS operations in an urban environment may suffer from navigation solution outages due to shadowing of GNSS signals from objects and buildings or deteriorated positioning performance due to multipath and bad available satellite geometry. An example of the predicted availability and accuracy performance using both GPS and Galileo is discussed in [1]. In this paper, we model the north campus of TU Berlin with its buildings as a set of GML-objects with multiple urban canyons in between (Fig. 1, right) and analyze the effects on the GNSS-constellation visibility both for layer grids, recorded tracks and a fictitious UAS approach path. Due to the limited availability and deteriorated navigation performance, urban environments are often referred to as GNSS-challenged environments. To improve availability and guarantee continuity of service in these environments, GNSS can be integrated with an IMU [2] or improved by increasing its sensitivity by using external data sources (i.e., assisted GPS). This integration strategy is successful in many cases but does not cover all possible scenarios. To enable operation of UAS at any time in any environment, a navigation capability is required that is robust and not solely dependent on GNSS. Furthermore, the trajectory, thus the flight plan of the UAS, should be optimized prior to commencing the flight applying a multi-criteria optimization algorithm that takes not only GNSS availability and geometry into account, but also factors like traffic separation assurance, (time) efficiency, minimum noise impact, and meteorological conditions during enroute, take-off and landing phases of urban flight.
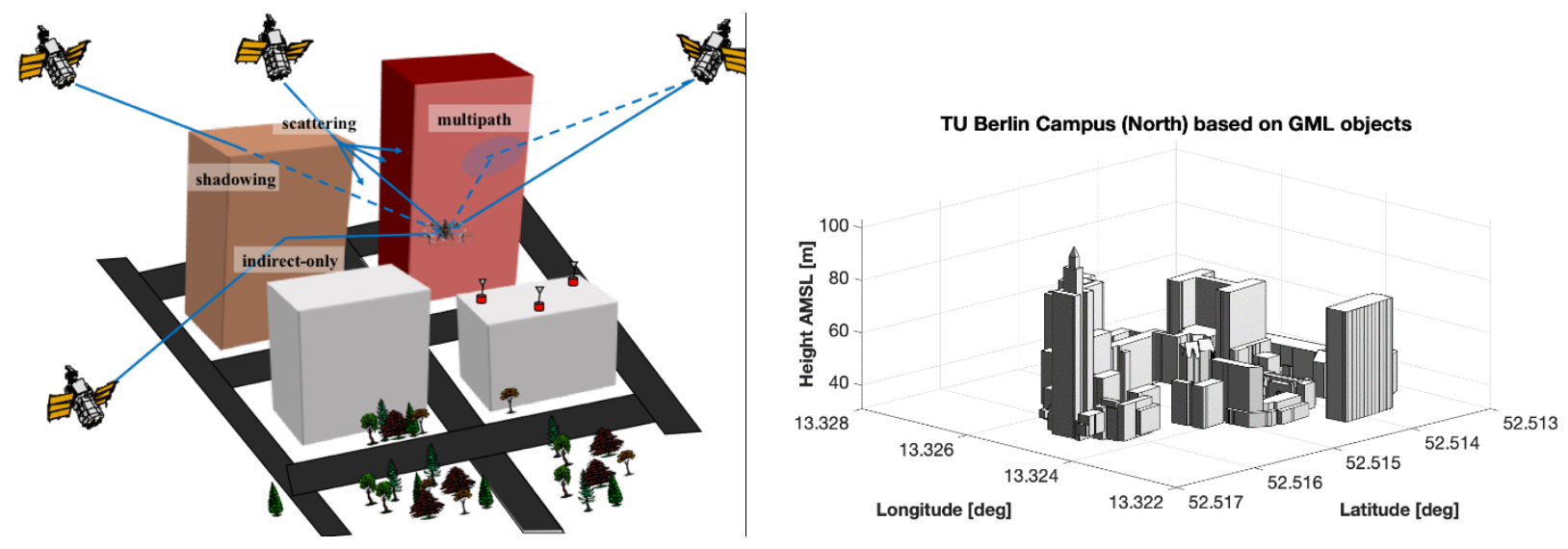

Fig. 1: Challenging GNSS environment due to signal shadowing and multipath

This paper describes a method that not only uses GNSS to determine the user position inflight, but integrates GNSS with inertial data, laser-based sensors, vision sensors, range radios and a map of the urban environment to obtain a reliable (i.e. assured) position estimate with respect to the usual navigation performance parameters (accuracy, integrity, availability, and continuity). In order to estimate the need for additional means of navigation during flight (strategic level), we present results of an extensive GPS availability analysis for a fictitious UAS mission scenario that 
can be performed prior to the sUAS mission and uses urban objects data (geographic markup language, GML) and ephemeris data of the GPS. Later, this model can be extended with other attributes as well such as traffic, noise impact, weather, etc.

The remainder of this paper is structured as follows: In section 2, the basic framework levels for assured multi-mode navigation of sUAS will be described and the current scientific state both for sUAS trajectory optimization and multi-mode navigation is examined. In section 3, we describe the methodology for GNSS availability and performance analysis in urban areas and outline the conceptional approach for multi-mode navigation during all phases of flight. Section 4 shows the results of the GNSS availability and performance analysis and results of using imagery as primary navigation source, where GNSS is only sparsely available.

\section{BACKGROUND}

Assured multi-mode navigation requires the application of different navigation methods inflight and during different flight phases (tactical level), but also flight planning prior to the flight (strategic level), where the flight trajectory (flight track and vertical path) is optimized using various criteria including GNSS availability and the aforementioned criteria (Fig. 2).

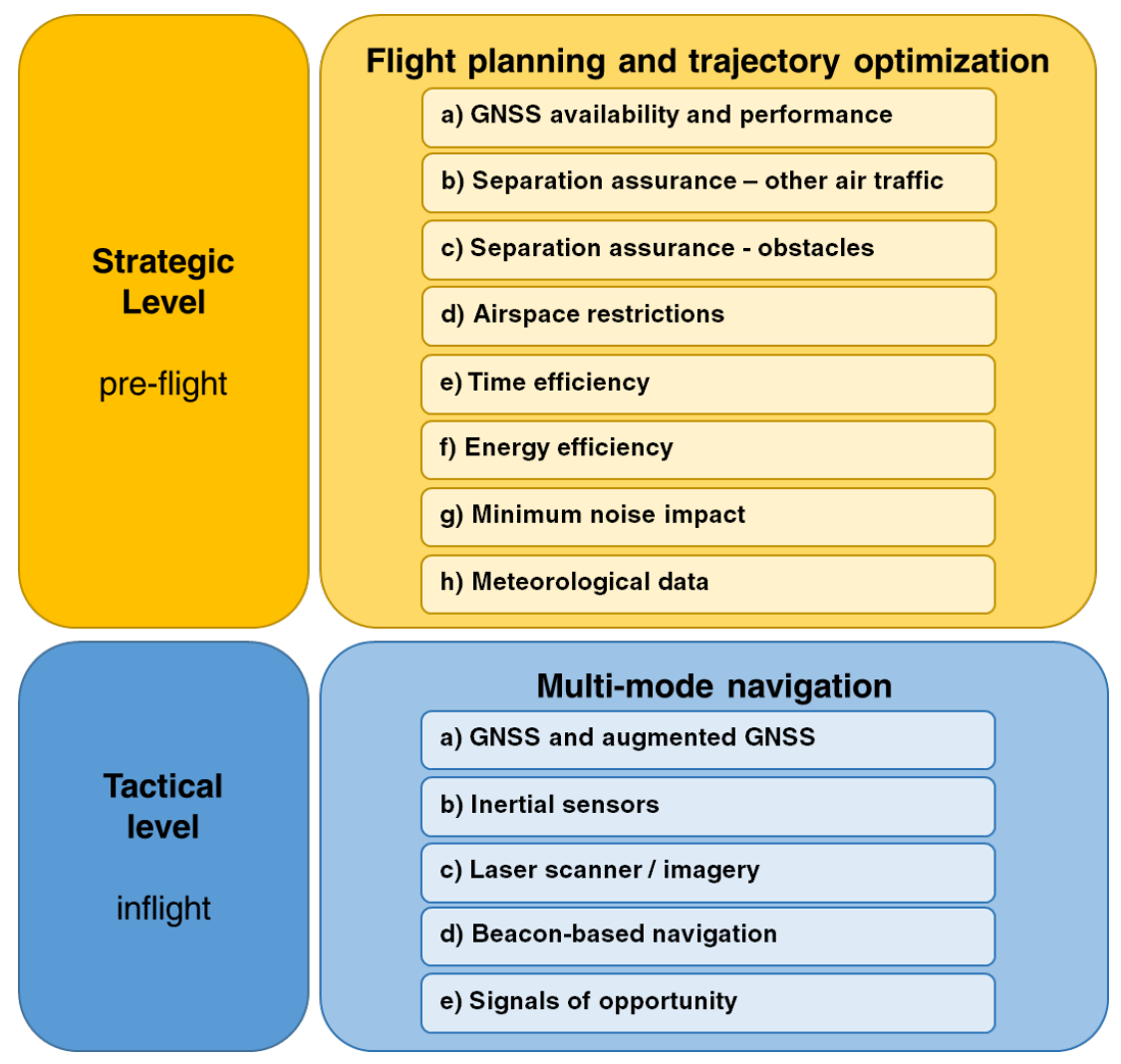

Fig. 2: Basic framework levels of assured multi-mode navigation of sUAS 


\subsection{Flight planning and trajectory optimization}

Trajectory optimization for small UAS (UAS) missions prior to the flight as part of the flight planning process will be invaluable for enabling UAS operations for specific application needs. Multi-criteria trajectory optimization solutions are widely available for the commercial (manned) aviation sector, where the flight trajectory of single flights and air traffic volumes is optimized in 4D for safety (e.g. flight separation), time and fuel efficiency, and environmental impact [3]. Vilardaga et al. focus in [4] and their earlier research work on the optimization of conflict-free trajectories in terminal maneuvering areas (TMA) around airports. Their proposed optimization framework for conflict-free trajectories enables an increase of capacity and fuel-efficiency for departing and arriving air traffic within the TMA, by implementing a continuous and multiphase optimal control (non-linear) problem with a set of finite decision variables. The algorithm continuously monitors the optimal trajectory based on the surrounding air traffic using ADS-B-in information to predict 4D-position of potential intruders. Both research outcomes are promising for application to UAS Traffic management (UTM) trajectory optimization.

Like trajectory optimization for the commercial and manned aviation sector, trajectory optimization for UAS traffic has been recently studied extensively in the scientific literature. This is expected given the broad spectrum of UAS applications on one hand and very limited performance and system characteristics of UAS (small size, limited weight, payload and limited power) on the other requiring an elaborated mission planning to guarantee most optimized flight trajectories.

Pérez-Carabaza et al. [5] present a Minimum-Time-Search (MTS) planner, which enables UAS to complete search missions for life boats with minimum risk of collision or communication loss with the ground operator. The underlying MTS-algorithm helps search and rescue (SAR) missions by minimizing the target detection time. In the field of UAS-assisted cellular networks and for UASbased Internet of Things (IOT) Chowdhury et al. [6] demonstrate a method to identify the optimal trajectory for UAS to improve the coverage of a terrestrial cellular network under time constraints by solving the optimization problem using a dynamic programming technique. In [7] Causa et al. present a cost-driven optimization algorithm for path planning of Multi-UAS missions in urban scenarios with heterogenous GNSS coverage and performance. They suggest a cooperative navigation concept for challenging environments in urban areas, where navigation tasks are distributed among the UAS swarm.

Though the application areas of the above research are very specific, all of them show the wide range of possibilities for trajectory optimization for UAS flight planning. In view of this and other research in this field, we are developing a mission planning framework for sUAS, where multiple optimization criteria are applied during flight planning to identify the most-suitable mission trajectory. Based on the meta study performed by Coutinho et al. in [8] we identified 8 main criteria on the strategic level (a-h) that will be addressed in our mission planning framework (see Fig. 2). In the following sections 3 and 4 , we will focus on criteria (a). Criteria (b) through (h) will be the focus in future papers. The sections describe the methodology to check the GNSS availability and performance pre-flight and determine of alternatives or augmentations to GNSS-based navigation are required along the flight path in a challenging environment. It will furthermore enable the selection of the trajectory that optimizes the best GNSS coverage and performance, to which the 
remaining criteria will be added. This work will be based in part on previous research work from the authors [4].

\subsection{Multi-mode navigation}

In case GNSS by itself is not available or sufficiently deteriorated alternative navigation methods can be used on a UAS including (i) the integration of laser scanners and/or imagery with an inertial sensor [9], (ii) the use of signals of opportunity [9], (iii) and beacon-based navigation (e.g. pseudolites, ultra-wideband (UWB) beacons, WiFi, cell-towers, terrestrial beacons) [9]. The methods under (i) typically exploit the observation of features in the environment using laser range scanners and imagery. With respect to (i), challenging environments are divided into two types of environments according to [11]: (a) the "structured" environment characterized by well-defined parameters such as predictable ceiling heights, room/corridor shapes/sizes, and standard building materials, (b) and the "unstructured" environment characterized by irregular dimensions and rough surfaces. Navigation in the former is referred to as structured environment navigation (SEN) and includes navigation for small UAS operation in urban and indoor environments. Navigation in the latter is referred to as probabilistic environment navigation (PEN) and includes navigation in forest, caves and old mines.

\section{METHODOLOGY}

\subsection{GNSS availability and performance analysis}

The analysis of GNSS availability in an urban environment based on satellite geometry and shadowing effects have been investigated in a variety of papers in recent years. Many studies are using the predicted receiver position, satellite constellation data of an observation epoch and an object database to calculate and proof the line-of-sight (LOS) to each available satellite in the hemisphere. Once the visible satellites are identified the dilution of precision (DOP) with its specific values is calculated as a measure of the satellite geometry for the receiver location to describe the effects on the position accuracy [12][13][14].

Our predictive GNSS availability and performance analysis follows a similar but segregated approach, as shown in Fig. 3. The result of our analysis is a grid both for the number of visible satellites and DOP-values (horizontal dilution of precision, HDOP; vertical dilution of precision, VDOP; position dilution of precision, PDOP; geometric dilution of precision, GDOP; time dilution of precision, TDOP) for any urban environment. We will visualize and discuss our results as heatmap in section 4 for the example of TU Berlins' north campus with high buildings (> $40 \mathrm{~m}$ above ground level) and urban canyons in between. In future implementations, we envision the inclusion of horizontal and vertical protection levels (HPL and VPL) to support urban performancebased navigation (PBN). 


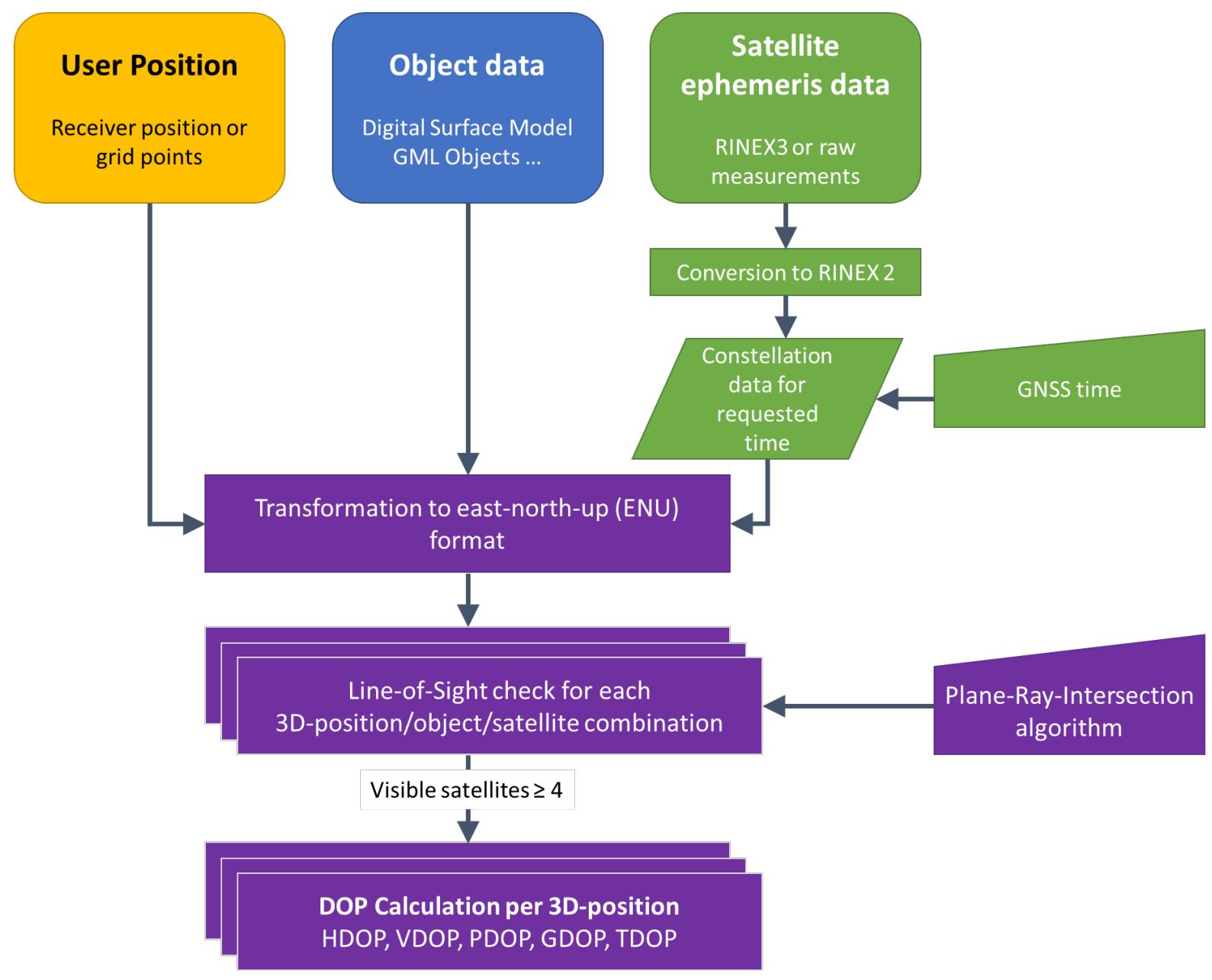

Fig. 3: DOP-calculation workflow

\subsubsection{Data preparation}

Our DOP-calculation workflow, illustrated and simplified in Fig. 3, follows the approach of [14]. Here, the user position can be defined either by a set of grid points for the area, where the UAS will be operated, or by a specific point of interest. The object data for urban areas is most commonly available via public sources. We used GML-defined objects, where significant corners and edges of buildings are defined in 3D, either in the WGS84- or UTM-reference system (like the user position). GNSS satellite constellation data (GPS, GALILEO or GLONASS), available as either hourly or daily file, is retrieved from the ESA IGS GDC Service (European Space Agency - International GNSS Service - Global Data Center) ${ }^{4}$ as RINEX3-file, which is then converted to the older RINEX2 format for data handling issues. The data conversion is done by the "RINEX GNSS Data Conversion and Manipulation Toolbox" available from the German Research Center for Geosciences (GFZ) enabling both RINEX3 to RINEX2 conversion or vice versa. [15] After adding a specific GPS time, which is required to select the correct time frame for GNSS geometry analysis, we get the number and position of the visible satellites for our hemisphere in earth-centered, earthfixed (ECEF)-reference system. For practical reasons and enable the DOP-calculation, we convert

4 Available at: https://gssc.esa.int/activities/igs-global-data-center/ (Last access: 28/11/2019) 
all position data (user position, object data, satellite constellation data) into local east-north-up (ENU) reference system, implementing sub-transformation algorithms from UTM33U to WGS84, WGS84 to ECEF and ECEF to ENU.

\subsubsection{Line-of-Sight check}

The GNSS availability analysis is performed as direct line-of-sight (LOS) check between each grid/user point and each satellite. Using an algebra-based plane-line-intersection algorithm we determine whether the LOS-vector between grid/user point $P 0_{i j}$ and satellite vehicle $S V_{n}$ penetrates any vertical object surface $k_{m}$ in between [1]. Fig. 4 illustrates the function principle of this well-known algorithm: Here, we subdivide each surface in a finite number of triangles, calculate the unit vector $\vec{n}$ for each triangle and apply the plane-line-intersection-algorithm. The algorithm checks, whether i) the vector is parallel to $\vec{n}$ (no intersection), ii) intersection point lies within the triangle plane, but not within the triangle boundary $\mathrm{ABC}$ or iii) intersection point $I_{k, m, A B C}$ lies within the triangle $A B C$, which indicates a blocked LOS. In case of (iii) the algorithm stops immediately and proceeds to the next satellite or user position. To accelerate the processing time, we eliminate all horizontal surfaces of the objects, because in case of a blocked LOS the vector would always hit a vertical surface first. The identification of a horizontal surface within one object is done by calculate the standard deviation $s_{k, m, z}$ along all z-coordinates. If $s_{k, m, z}<0.01$, we define the object surface as horizontal.
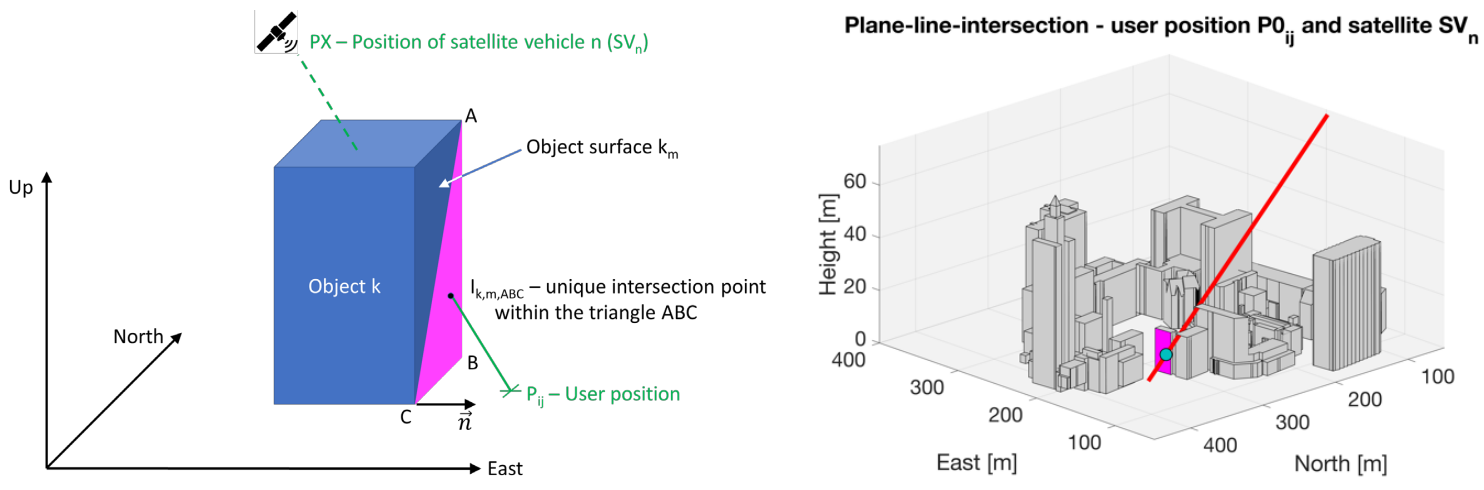

Fig. 4: Plane-line-intersection principle (left) and unique intersection point for LOS-vector and object (right)

The algorithm is performed for every 3D-grid position - satellite combination. The output of the algorithm is both the number of visible satellites per 3D-grid/user position as well as the respective satellite vehicle number to enable satellite geometry analysis (see following subchapter) for a specific grid/user point.

\subsubsection{Calculation of dilution-of-precision (DOP) values}

After the LOS-analysis for a given grid in an urban environment, we study the achievable accuracy, thus uncertainty, for any grid point in an urban environment by calculating the characteristic dilution-of-precision (DOP) values VDOP (1D), HDOP (2D) and PDOP (3D). The DOP-calculation requires at least 4 visible satellites, to solve $x, y, z$ and the receiver clock bias. The DOP values are built up from the characteristic covariance matrix $G$ of the LOS-observations for a specific grid point. The covariance matrix $G$ depends on the satellite geometry and amount of visible satellite 
and the DOP values are derived from the trace elements of $G$, as described very detailed in Kaplan et al. [16]:

$$
\begin{aligned}
& \begin{array}{lllll}
\sigma_{x}^{2} & \sigma_{x y} & \sigma_{x z} & \sigma_{x t}
\end{array} \\
& G=\begin{array}{cccc}
\sigma_{x y} & \sigma_{y}^{2} & \sigma_{x z} & \sigma_{y t} \\
\sigma_{x z} & \sigma_{y z} & \sigma_{z}^{2} & \sigma_{z t}
\end{array} \\
& \begin{array}{llll}
\sigma_{x t} & \sigma_{y t} & \sigma_{z t} & \sigma_{t}^{2}
\end{array} \\
& G=\left(H^{T} \cdot H\right)^{-1} \text { with } H=\left[\begin{array}{c}
H_{1} \\
\vdots \\
H_{n}
\end{array}\right] \text { where } H_{n}=\left[\frac{x_{n}-x}{r_{n}}, \frac{y_{n}-y}{r_{n}}, \frac{z_{n}-z}{r_{n}},-1\right] \\
& \text { and } r_{n}=\sqrt{\left(x_{n}-x\right)^{2}+\left(y_{n}-y\right)^{2}+\left(z_{n}-z\right)^{2}}
\end{aligned}
$$

$H_{n}$ is defined as the unit vector from the grid position to the satellite $\mathrm{n}$, where $x_{n}, y_{n}$ and $z_{n}$ are the ENU-position of the satellite $\mathrm{n}, x, y$ and $z$ are the grid position (ENU) and $r_{n}$ represents the range between the grid position and satellite $n$. The DOP-values are calculated for each grid point as follows using the diagonal elements of G (not including GDOP and TDOP) [16]:

$$
\begin{aligned}
& \operatorname{VDOP}(1 D)=\sqrt{\sigma_{z}^{2}}=\sqrt{\sigma_{u p}^{2}} \\
& \operatorname{HDOP}(2 D)=\sqrt{\sigma_{x}^{2}+\sigma_{y}^{2}}=\sqrt{\sigma_{\text {east }}^{2}+\sigma_{\text {north }}^{2}} \\
& \operatorname{PDOP}(3 D)=\sqrt{\sigma_{x}^{2}+\sigma_{y}^{2}+\sigma_{z}^{2}}=\sqrt{\sigma_{\text {east }}^{2}+\sigma_{\text {north }}^{2}+\sigma_{u p}^{2}}
\end{aligned}
$$

Using the formulas (1)-(6), we calculate the DOP-values for the north campus of TU Berlin for a specific satellite constellation and observation epoch. The results and discussion are shown in section 4 will enable the prediction of the most-suitable flight path with best GNSS-availability. Missing in the simulation so far are the effects of multipath, scattering, and indirect only GNSS paths. These effects can not be ignored and will be addressed in furture work by the authors.

\subsubsection{Other than GNSS availability optimization criteria}

Optimization algorithms for aforementioned criteria are currently studied and developed, based on the presented research work of the authors and others (see section 2). Preliminary results are published soon by the authors.

\subsection{Conceptional approach for multi-mode navigation inflight}

The initial operational scenario for the proposed method of multi-mode navigation is shown in Fig. 5. The operational scenario will base on the outcome of the flight planning and trajectory optimization results to estimate the need for alternative means of navigation.

Vertical take-off takes place in an open area with good GNSS visibility and the navigation solution is based on SBAS or dual-frequency (E1, E5) GNSS integrated with inertial (1). During the next two phases (2) and (3), the sUAS will transition from its take-off location over or through an urban 
environment to the destination following the approved flight plan from the U-space/UTM service provider. During this phase-of-flight, the sUAS will continuously determine its actual navigation performance (ANP) and compare it to the Required Navigation Performance (RNP) associated with the flight plan. The method further assesses the effect (threat) imposed by the urban environment on the GNSS signal (e.g. multipath, reflection only, scattering). For example, when flying over the urban landscape (2), GNSS is minimally affected, but as soon as the sUAS operates in the urban canyons (3), the method must assess what alternative positioning sensor mode is available (i.e. laser scanners, vision cameras, or range radio beacons) and switch to a different integration mechanization (i.e. filter).

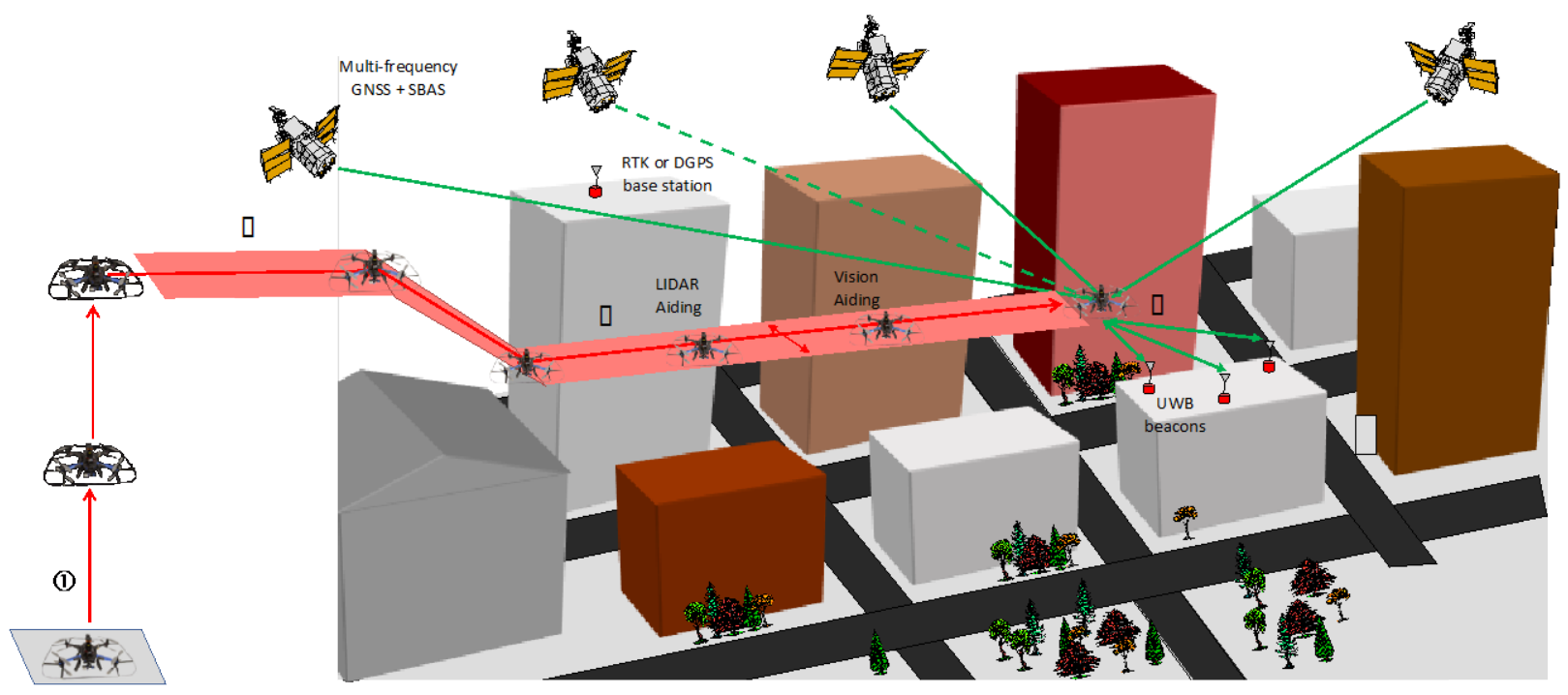

Fig. 5: Navigation mode switching in an urban environment.

Note that when the sUAS transitions from (2) to (3), the sensors perceive the environment and use the environment or feature in the environment to "help" navigate. In addition to supporting the navigation function, these sensors may (and will) also be used for detect/sense and avoid.

The block diagram of the navigation filter is shown in Fig. 6. On the left side are the sensors that are considered in the position estimator (i.e. filter mechanization). After pre-processing, the measurements are screened for suitability and quality (threat and observability analysis block) and used to determine the position of the sUAS (in the multi-mode filter block). Furthermore, the filter residuals are monitored to detect possible failures that made it through the screening process. This position is then fed back to previous block for geo-referencing of the laser and camera data and as an input to the urban database for the measurement screen process. The geo-references laser and camera data can then be used in the detect and avoid function. This concept bases, in part, on work performed by the authors [17][18]. 


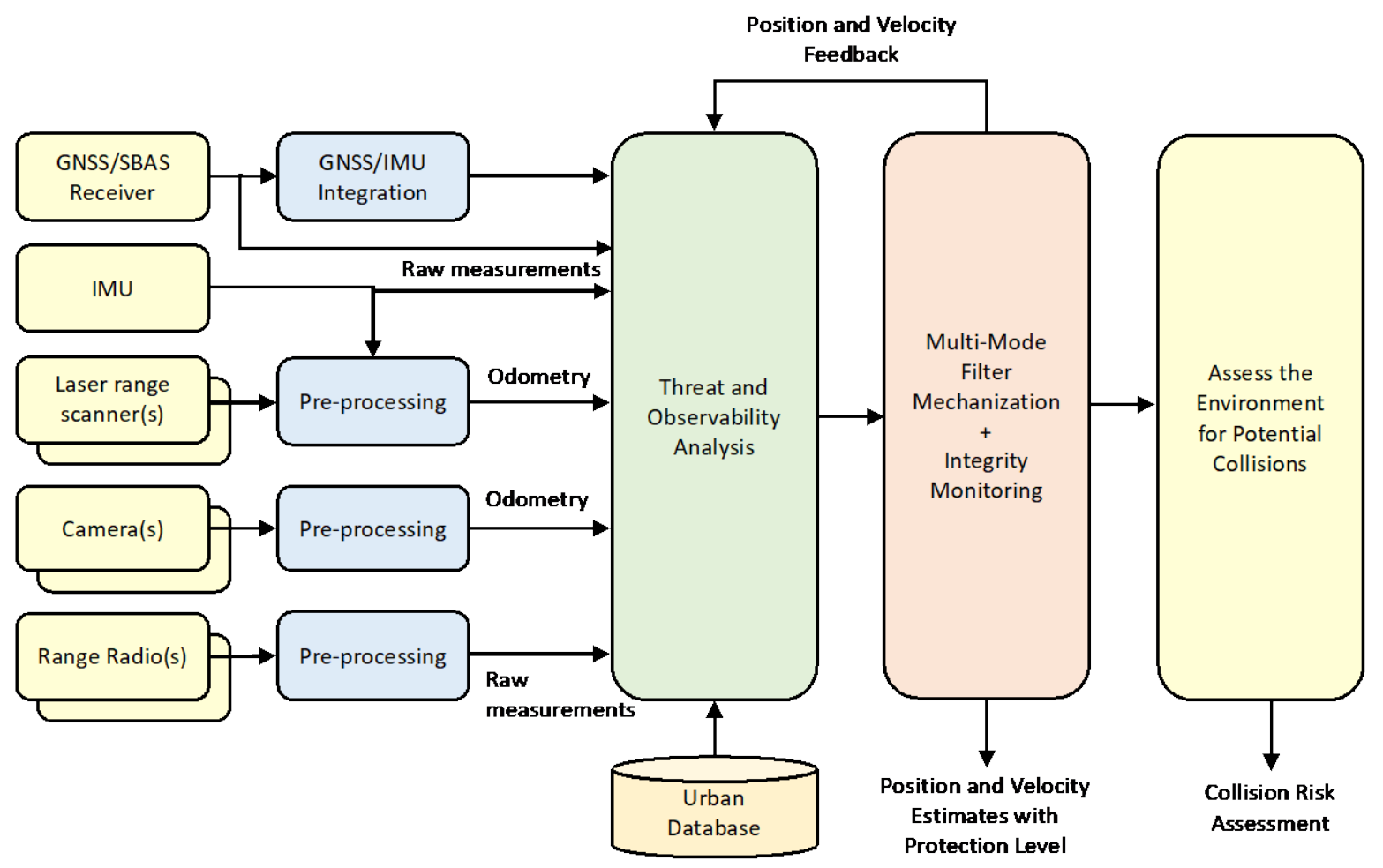

Fig. 6: High-level functional block diagram of the multi-mode navigation function.

Fig. 7. Shows an example of a sUAS equipped with laser scanners (Hokuyo), laser altimeter (WASP LRF200), a camera, a GNSS (uBlox F9T timing board) and an IMU setup for research purposes and planned for future validation missions of the availability tool.

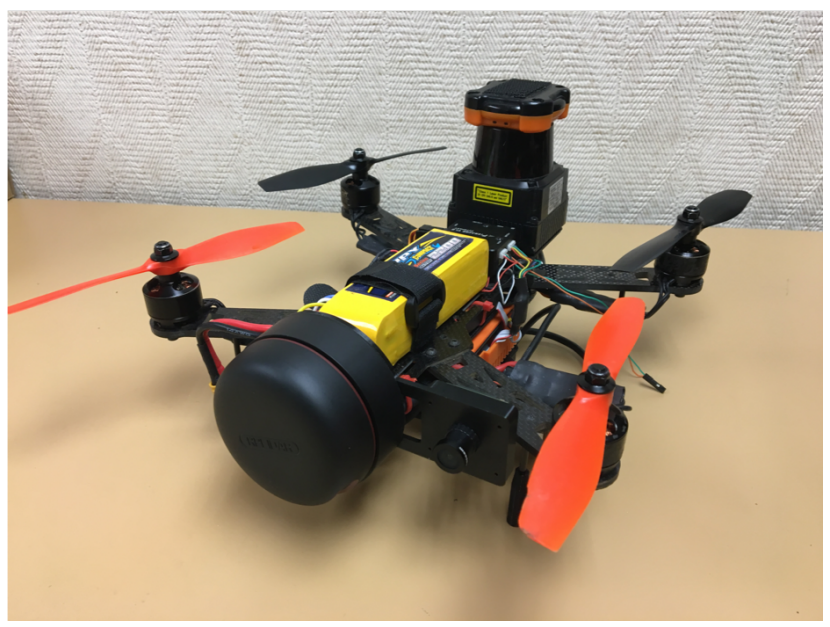

Fig. 7: Example sUAS research platform with multi-mode navigation capability 


\section{RESULTS}

\subsection{GNSS availability analysis}

In the following section we show the results of the GNSS availability analysis for the urban environment of the TU Berlin north campus with characteristic urban canyons. The heatmaps show a static observation epoch for a specific GPS-satellite constellation. However, the algorithm is able to compute day-time dependent satellite constellation data, based on the RINEX2-file as data input.

All grid-based results are defined by a 4D-matrix, containing $x, y, z$-coordinates and the calculated values for the amount of visible satellites $\left(\mathrm{SV}_{\text {vis }}\right)$ or DOP-values as a $4^{\text {th }}$ dimension. Using a fifth dimension for each 4D-grid point (e.g. costs), we can apply, for example, a Dijkstra-based pathfinding algorithm to optimize a given trajectory for the best GNSS availability. High GNSS availability (high number of visible satellites, low DOP-values) will result in low costs, thus the algorithm will determine the path with lowest costs.

\subsubsection{Heatmap of visible GPS satellites}
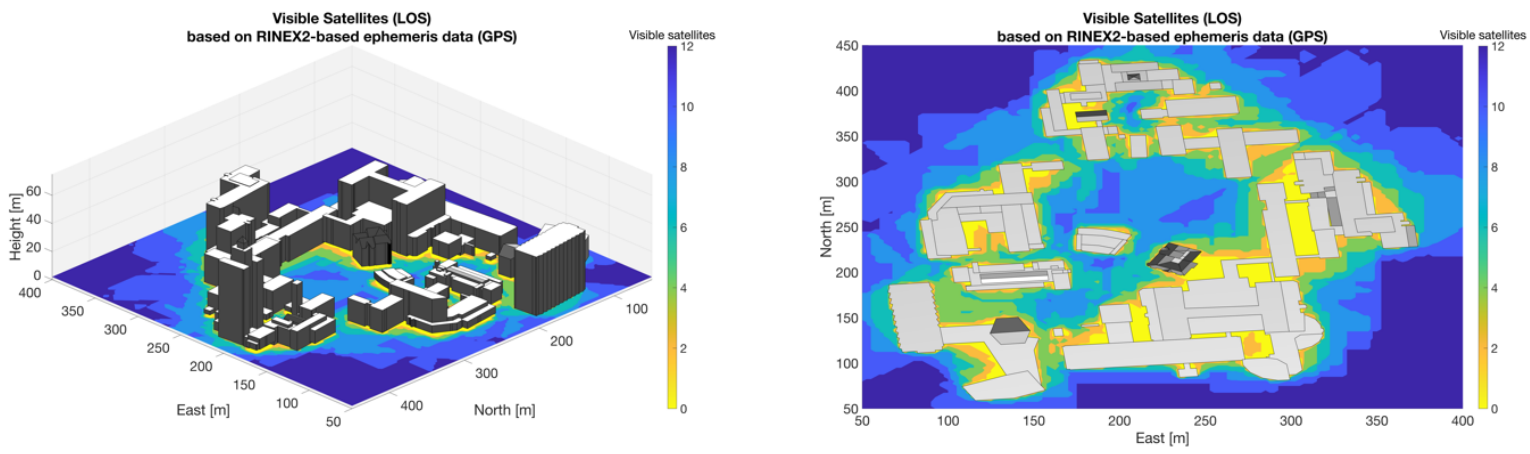

Fig. 8: Heatmap of visible satellites for TU Berlins' north campus based on line-of-sight calculation

Fig. 8 shows the number of available satellites $S_{\text {vis }}$ calculated according the described methodology in section 3.1. For a specific date and GPS time, 12 satellites where visible for our hemisphere. Clearly visible are GPS-signal shadowing effects close to and between buildings, where less (green/orange) or no visible satellites (yellow) were available. For areas with $\mathrm{SV}_{\text {vis }}<4$ (green, orange and yellow colors) GPS-based 3D-navigation is not possible, thus multi-mode navigation has to be applied to ensure safe navigation.

\subsubsection{Comparison of calculated and measured amount of visible satellites}

A uBlox ZED-F9P Multiband RTK GPS receiver (Ardusimple) was used to log the raw data, 3D position and used satellites for positioning along a trajectory over the campus and compared the results with the calculated number. The results are shown in Fig. 9. The measured number of satellites was besides a single track point always higher than our calculation, with a mean difference of +3.5 satellites and a standard deviation of 2.1 between the measured and the calculated number. In general, the prediction of visible satellites looks reasonable, however in areas with high shadowing the deviation to the measurement is higher than average. The difference in the 
results is most likely due to multipath effects or the reception of reflection-only GNSS satellite signals, which is not recognized in the algorithm so far. Furthermore, our logged track around the building was in the north-west part very close $(<2 \mathrm{~m})$ to the building. As the GPS positioning might not very accurate in that area the track is supposed to be within parts of the building, which means not any LOS with satellite vehicles.
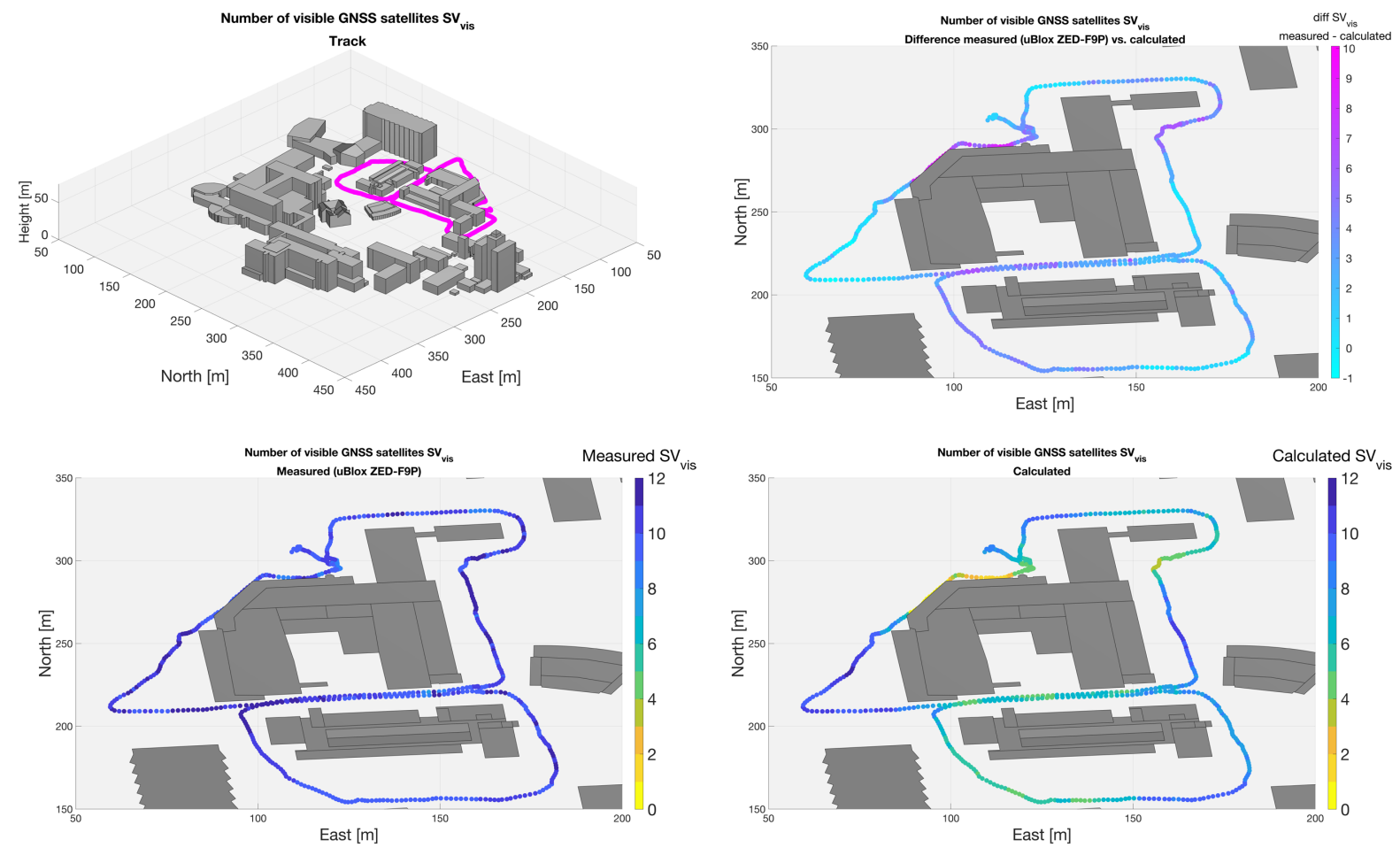

Fig. 9: Comparison of calculated and measured amount of visible satellites

\subsubsection{Heatmaps of DOP values}

The characteristic DOP values VDOP (1D), HDOP (2D) and PDOP (3D) are calculated along a grid (spacing $2.5 \mathrm{~m}$ ) according (1)-(6) and results are illustrated in Fig. 10. White areas (close to or between buildings) indicate no sufficient GNSS satellite availability (SV vis $<4$ ), thus no DOP calculation is possible. Based on the current (static) GPS-constellation and -geometry we identify areas close to fronts of buildings with less corners or protrusions, where comparable low DOPvalues still are achieved, while buildings with unsteady fronts show larger areas of poor DOPvalues (>10). As Fig. 10 shows the results for ground level only, higher altitudes will produce better DOP with qualitative lower values, while signal shadowing effects due to buildings in between will become less. In addition, the DOP-heatmaps show clearly areas, where UAS-missions have to rely on multi-mode navigation: Bossler et al. [19] define an acceptable PDOP value for navigation between 3 and 6 . We will use the lower end (PDOP $<3$ ) as the limit, where multi-mode navigation sources besides GNSS have to be used for navigation of UAS. Results showing a PDOP $<3$ are given in the lower right heatmap of Fig. 10. Now, to enable some form of PBN in future urban navigation scenarios, future analyses must include HPL and VPL calculations. However, for those multipath, reflection only, and scattering models must be included and integrated strategies with alternative navigation sensors identified (e.g. laser range scanners and cameras). 
The effects of the satellite geometry for that static observation are clearly visible, as we can identify pre-dominant shadowing in south-west/north-east direction.
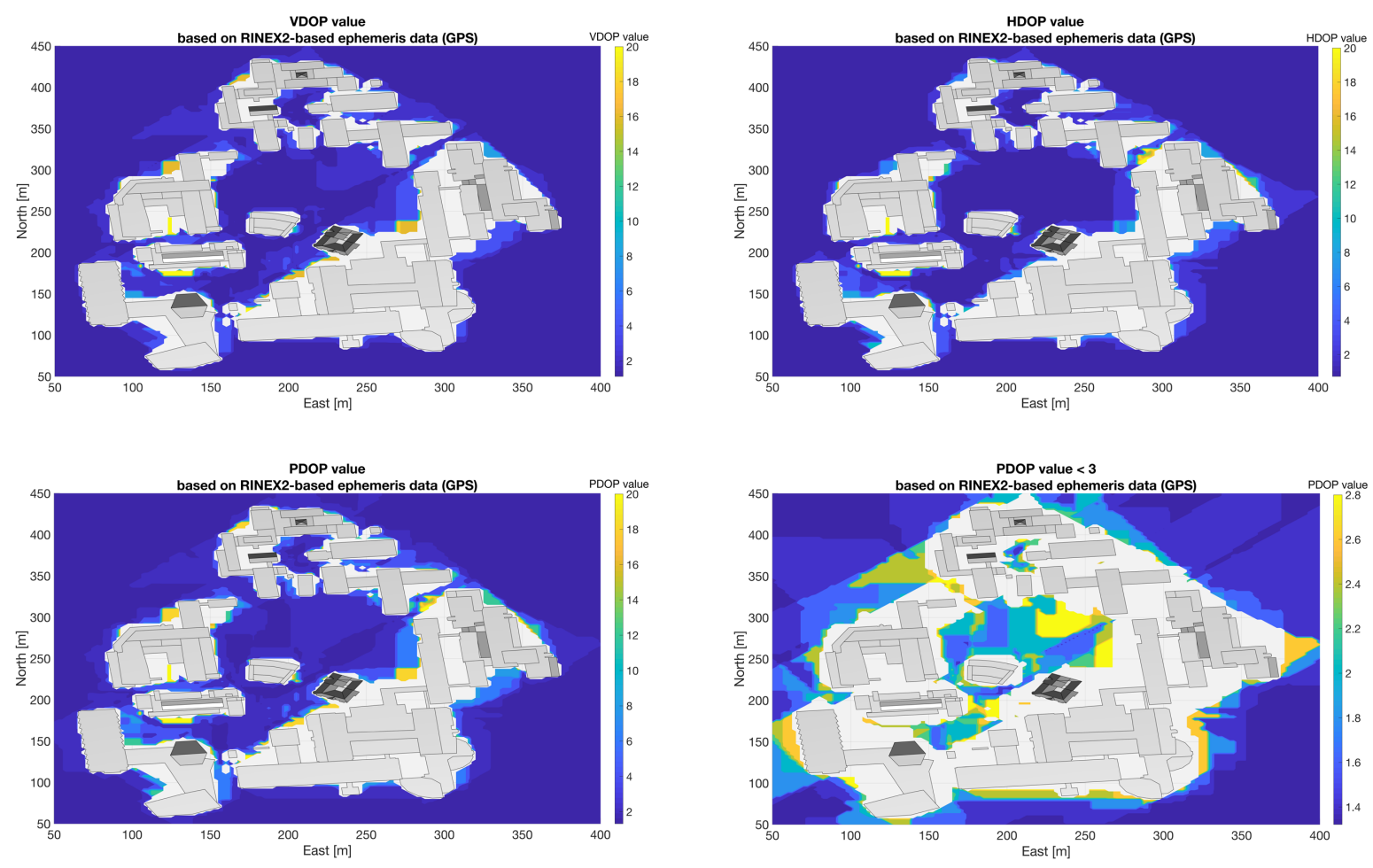

Fig. 10: VDOP (top-left), HDOP (top-right), PDOP(bottom-left), PDOP<3 (bottom-right) of TU Berlins' north campus at ground level

\subsubsection{Visualization of a fictitious UAS approach path as outlook for UTM traffic optimiza- tion}

The prediction of the GNSS availability along a fictitious UAS approach path is shown in Fig. 11 with a specific and static GPS-constellation. Although the full approach trajectory can be performed with a PDOP $<2$ until landing, the flight phase below $10 \mathrm{~m}$ above ground level (yellow marked) must be flown using multi-mode navigation to meet the increased required navigation performance during the final approach segment. Offering this information during UAS mission planning to the operator will give a better overview about the predicted GNSS performance in urban environments, and the ability for the operator to access if his UAS is sufficiently equipped to perform the procedure 


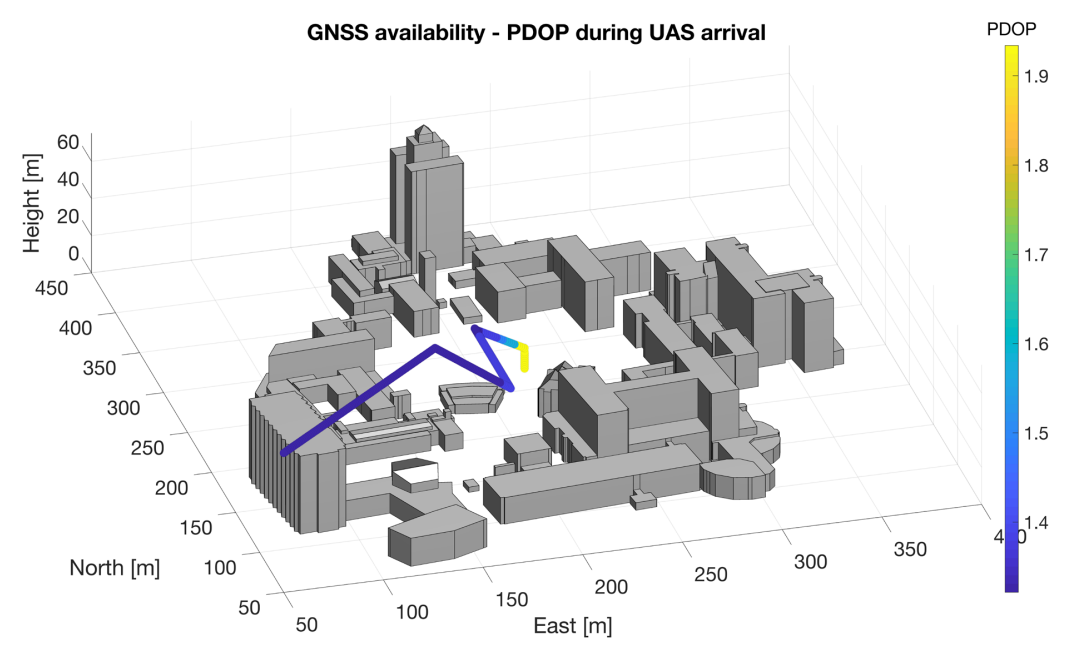

Fig. 11: PDOP-calculation for UAS approach path

\subsection{Multi-mode navigation}

As shown in Figure 6, laser range scanners, vision systems as well as a laser or radar altimeter are good candidates for integration with GNSS in addition to an inertial measurement unit, to provide continuity of the navigation function in areas where the GNSS-only solution is not sufficient. These integration strategies have been explained in detail in previous work by the authors [17][18][20]. Some preliminary outputs are shown in Fig. 12 and Fig. 13 for a flight right outside the Stocker Center engineering building at Ohio University where GNSS is only sparsely available.

The referenced scans associated with the horizontal and vertical laser range scanners can be observed clearly super-imposed on the 2D occupancy grid provided by the 2D Simultaneous Localization and Mapping (SLAM) component of the multi-mode filter mechanization. The larger coverage of the horizontal scans in the vertical direction can be attributed to the natural up-and-down motion of the drone under manual operation. This motion can be introduced in the flight plan when operating the UAS if necessary.

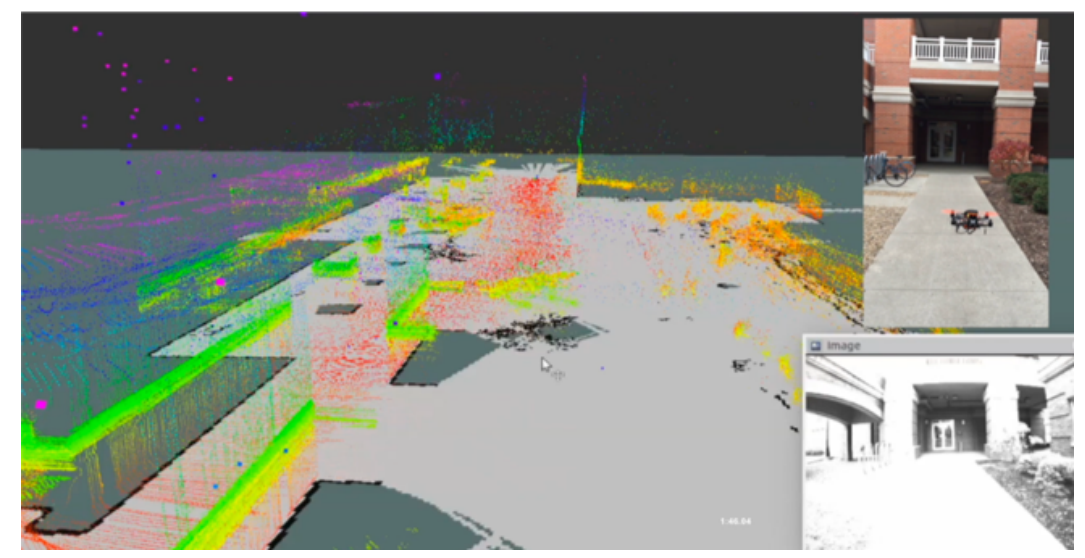

Fig. 12: Preliminary flight test results; navigation performance is evaluated by using the trajectory to make a map and comparing the map to the "real" map of the environment. 
Figure 13 shows an example of a map (point cloud) generated with vision-based navigator part of the multi-mode filter mechanization using DSO (direct, sparse odometry) as the visual odometer [21]. One can clearly observe the numerous features within the field of view. Unlike laser-based mapping, the vision-based solution has extent in the horizontal and vertical direction every frame due to its instantaneous field-of-view (FoV).

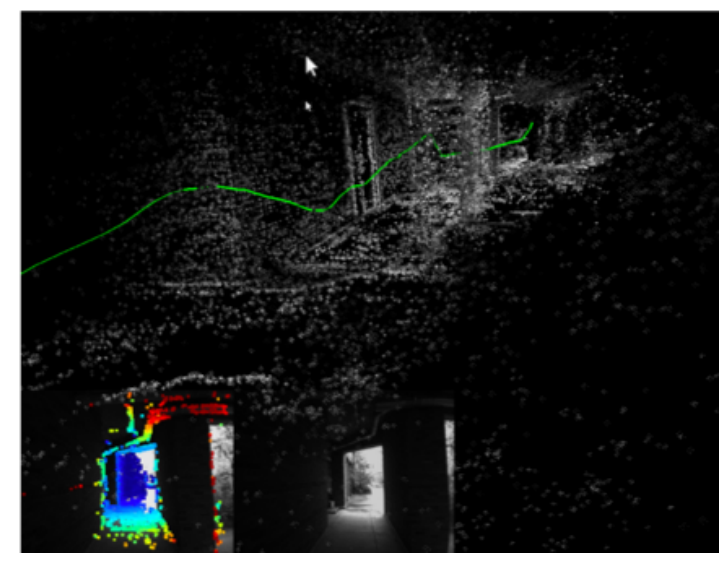

Fig. 13: Results based on using the camera images as a visual odometer (using DSO); navigation performance is evaluated by using the trajectory to make a map and comparing the map to the "real" map of the environment.

\section{CONCLUSION}

This paper discusses the development of a framework to enable and ensure multi-mode navigation for sUAS navigation in urban environments. The framework consists of two main stages: Flight planning and trajectory optimization (strategic level, preflight) and application of a multi-mode navigation concept inflight (tactical level). The trajectory optimization follows a multi-criteria approach, where we presented results for GNSS availability analysis in an urban environment. Based on the GNSS analysis and our presented algorithm, the operator will be able i) to determine the GNSS performance along the intended flight trajectory, ii) optimize the trajectory to meet GNSS-performance requirements and iii) select suitable multi-mode navigation measures besides GNSS to navigate in challenging environments, e.g. urban canyons with sparse or no GNSS availability. During the various phases of sUAS flight, the proposed method for multi-mode navigation uses the urban database, the output of the integrity monitor and various other metrics to select which measurements from SBAS GNSS, the IMU, the laser scanners, the cameras and range radios should be used in the filter that computes the sUAS position. Furthermore, the geo-references laser and camera data can be used to support the detect and avoid function to assess the risk of collisions with buildings or other stationary and dynamic objects. Currently, the GNSS availability tool only supports GPS and loss of direct line-of-sight. Future upgrades include other constellations as well as better models for multipath, scattering and reflection-only receptions. Furthermore, models are being developed for the expected performance (and, thus, availability) of laser-range scanner and vision-aiding strategies. 


\section{REFERENCES}

[1] Kleijer, F., Odijk, D., Verbree, E.. (2008). Prediction of GNSS Availability and Accuracy in Urban Environments - Case Study Schiphol Airport, Book chapter in Location-based Services and Cartography II.

[2] Farrell, J. L. (2007). GNSS Aided Navigation \& Tracking - Inertially Augmented or Autonomous, American Literary Press.

[3] Rosenow, J., Förster, S., Lindner, M., \& Fricke, H. (2017). Impact of multi-criteria optimized trajectories on European Air Traffic Density, efficiency and the environment. 12th USA/Europe Air Traffic Management $R$ and D Seminar, Seattle.

[4] Vilardaga, S., Prats, X., Duan, P., \& Uijt de Haag, M. (2014). Conflict free trajectory optimisation with target tracking and conformance monitoring. AIAA AVIATION 2014 -14th AIAA Aviation Technology, Integration, and Operations Conference, (June). https://doi.org/10.2514/6.2014-2022

[5] Pérez-Carabaza, S., Scherer, J., Rinner, B., López-Orozco, J. A., \& Besada-Portas, E. (2019). UAV trajectory optimization for Minimum Time Search with communication constraints and collision avoidance. Engineering Applications of Artificial Intelligence, 85, 357-371. https://doi.org/10.1016/i.engappai.2019.06.002

[6] Chowdhury, M. M. U., Bulut, E., \& Guvenc, I. (2019). Trajectory optimization in uav-assisted cellular networks under mission duration constraint. IEEE Radio and Wireless Symposium, RWS. https://doi.org/10.1109/RWS.2019.8714567

[7] Causa, F., Fasano, G., \& Grassi, M. (2018). Multi-UAV path planning for autonomous missions in mixed GNSS coverage scenarios. Sensors (Switzerland), 18(12). https://doi.org/10.3390/s18124188.

[8] Coutinho, W. P., Battarra, M., \& Fliege, J. (2018). The unmanned aerial vehicle routing and trajectory optimisation problem, a taxonomic review. Computers and Industrial Engineering, 120, 116-128. https://doi.org/10.1016/j.cie.2018.04.037

[9] Miller, M. M., Uijt de Haag, M., Soloviev, A., Veth, M. (2008). "Navigating in Difficult Environments: Alternatives to GPS - 1," Proceedings of the NATO RTO Lecture Series on "Low Cost Navigation Sensors and Integration Technology," SET-116, November 2008.

[10] Miller, M. M., Raquet, J., Uijt de Haag, M. (2008). "Navigating in Difficult Environments: Alternatives to GPS - 2," Proceedings of the NATO RTO Lecture Series on "Low Cost Navigation Sensors and Integration Technology," SET-116.

[11] Campbell, J. L. (2015). "Position, Navigation \& Time (PNT) for Contested/Denied Environments, AFRL Briefing," Navigation and Communications Branch, Sensors Directorate, AFRL.

[12] Suzuki, T., \& Kubo, N. (2015). Simulation of GNSS Satellite Availability in Urban Environments Using Google Earth. Proceedings of the ION 2015 Pacific PNT Meeting, 1069-1079.

[13] Lanagran-Soler, F., Vazquez, R., \& Arahal, M. R. (2015). A Matlab Educational GUI for Analysis of GNSS Coverage and Precision. IFAC-PapersOnLine, 48(29), 93-98. https://doi.org/10.1016/j.ifacol.2015.11.219

[14] Wang, L., Groves, P. D., \& Ziebart, M. K. (2012). Multi-constellation GNSS performance evaluation for urban canyons using large virtual reality city models. Journal of Navigation, 65(3), 459-476. https://doi.org/10.1017/S0373463312000082

[15] Nischan, T.. (2016): GFZRNX - RINEX GNSS Data Conversion and Manipulation Toolbox (Version 1.05). GFZ Data Services. http://doi.org/10.5880/GFZ.1.1.2016.002 
[16] Kaplan, E. and Hegarty C. (Editors) (2017). Understanding GPS/GNSS: Principles and Applications. Artech House.

[17] Dill, E. T., Uijt de Haag, M. (2016). "3D Multi-copter Navigation and Mapping using GPS, Inertial and LiDAR," NAVIGATION, Vol. 63.

[18] Dill, E. T., Young, S. D. \& Uijt de Haag, M. (2017). "Outdoor-to-Indoor UAV - GPS/Optical/Inertial Integration for 3D Navigation," GPS World, Vol. 28, Issue 10, pp. 20-26.

[19] Bossler, J. D., Campbell, J. B., McMaster, R. B., \& Rizos, C. (Eds.). (2010). Manual of Geospatial Science and Technology. CRC Press. https://doi.org/10.1201/9781420087345.

[20] Uijt de Haag, M., Robinson, J., Huff, J., Schultz, A. (2018). "Assessing Indoor Environments with sUAS through Real-Time Virtual Reality and Assured Navigation," Proceedings of the ION International Technical Meeting (ITM), Reston, VA, January 2018.

[21] Engel, J., Koltun, V., and Cremers, D. "Direct sparse odometry," IEEE Transactions on Pattern Analysis and Machine Intelligence. 